\title{
Synthesis of N-methyl-4-piperidone Curcumin Analogues and Their Cytotoxicity Activity against T47D Cell Lines
}

\author{
Yum Eryanti ${ }^{1{ }^{*},}$, Rudi Hendra ${ }^{1}$, Tati Herlina ${ }^{2}$, Adel Zamri ${ }^{1}$, and Unang Supratman ${ }^{2}$ \\ ${ }^{1}$ Laboratory of Synthesis Organic Chemistry, Department of Chemistry, Riau University, Pekanbaru 28293, Indonesia \\ ${ }^{2}$ Laboratory of Organic Chemistry, Department of Chemistry, Padjadjaran University, Bandung, Indonesia
}

Received April 19, 2017; Accepted August 2, 2017

\begin{abstract}
Three piperidone curcumin analogues (N-methyl-(3E,5E)-3,5-bis-(2-chlorobenzylidene)-4-piperidone (1), $N$ methyl-(3E,5E)-3,5-bis-(3-bromobenzylidene)-4-piperidone (2) and N-methyl-(3E,5E)-3,5-bis-(4-chlorobenzylidene)4-piperidone (3)) were synthesized from N-methyl-4-piperidone with halogenbenzaldehyde, 2-chlorobenzaldehyde, 3-bromobenzaldehyde and 4-chlorobenzaldehyde. The Claisen-Schmidt condensation reaction was used in alkali condition with combinatorial. All the compounds showed light yellow needle, light yellow powder, and yellow crystal form with percentage of yield 39, 66, and 40\%, respectively. All the structure compounds were confirmed by using UV, IR, ${ }^{13} \mathrm{C}-\mathrm{NMR},{ }^{1} \mathrm{H}-\mathrm{NMR}$ and MS. Apart from that, the cytotoxicity results against breast cancer cell (T47D) showed strong to moderate activity with the $I_{50}$ value 8,4 , and $45 \mu \mathrm{g} / \mathrm{mL}$, respectively.
\end{abstract}

Keywords: Curcumin; condensation; Claisen-Schmidt; cytotoxicity T47D

\section{ABSTRAK}

Tiga senyawa analog kurkumin turunan piperidon telah berhasil disintesis dari N-metil-4-piperidon dengan turunan halogenbenzaldehid, 2-klorobenzaldehid, 3-bromobenzaldehid dan 4-klorobenzaldehid melalui reaksi kondensasi Claisen-Schmidt dalam suasana basa secara kombinatorial. Analog kurkumin tersebut adalah $\mathrm{N}$-metil(3E,5E)-3,5-bis-(2,klorobenzaldehid)-4-piperidon (1), N-metil-(3E,5E)-3,5-bis-(3-bromobenzaldehid)-4-piperidon (2) dan N-metil-(3E,5E)-3,5-bis-(4-klorobenzaldehid)-4-piperidon (3). Ketiga senyawa tersebut memberikan bentuk dan warna berturut-turut kristal jarum kuning muda, serbuk kuning muda dan kristal kuning muda, rendemen 39, 66, dan 40. Semua senyawa tersebut telah dikonfirmasi strukturnya melalui analisis spektroskopi UV, IR, ${ }^{13} \mathrm{C}-\mathrm{NMR},{ }^{1} \mathrm{H}-\mathrm{NMR}$ dan MS. Uji aktivitas sitotoksisitas terhadap sel kanker payudara T47D memberikan nilai $I C_{50}$ sebesar 8, 4, dan 45 $\mu \mathrm{g} / \mathrm{mL}$ masing-masing untuk senyawa 1, 2, dan 3.

Kata Kunci: kurkumin; kondensasi; Claisen-Schmidt; sitotoksisitas T47D

\section{INTRODUCTION}

Curcumin is found in various types of Curcuma genera and is the major pigment contained in the turmeric plant (Curcuma longa). Curcumin, 4-demethoxy curcumin, bisdemethoxycurcumin, and dihydrocurcumin are found in turmeric [1]. Curcumin, a phenolic secondary metabolite, is believed to have antiinflammatory activity [2], antioxidant [3], antiviral, antiinfective and anti-allergic [4], as well as anti-HIV [5], and anti-cancer [6], and [7] properties.

Based on its biological activities, curcumin is attracting more interest as a model for new target compounds to be synthesized. Isolation of curcuminoids from natural materials found in small quantities is lowyield (3-5\% of the dry-weight), and the curcuminoids thus obtained possess limited structural variation. Indeed, this method presents an obstacle to optimize the function of curcumin [8]. Therefore, synthesis of curcumin derivatives might be conducted in the laboratory to obtain a reasonable amount of material as well as a wider variety of structures.

In general, curcumin can be made in a variety of methods one of which is through the condensation of an aldehyde with a ketone, either in acid or alkaline conditions. This method is known as aldol condensation reaction or, more specifically called the Claisen-Schmidt condensation reaction. Aldol condensation reaction are very popular and widely used in the formation of carbon-carbon bonds, because the reaction is simple, with readily available materials and is also known as environmentally friendly. Acid catalysts are commonly used in the aldol condensation reaction, such as dilute hydrochloric acid, while the base catalyzed conditions use sodium hydroxide, sodium methoxide, or lithium methoxide, either by used

* Corresponding author. Tel : +62--8127515110

DOI: $10.22146 /$ ijc.24174 
a solvent such as ethanol [9], or without solvent. Besides the above reasons, another attractive feature of this method is that it allows a combinatorial chemistry approach. Thus, this method can be used to create derivatives of curcumin with a variety of substituents on the benzene ring so that curcumin analog libraries can be rapidly produced. In continuation of our interest in the reaction of $\mathrm{N}$-methylpiperidin-4-one and (2clorobenzaldehyde, 3-bromobenzaldehyde and 4clorobenzaldehyde) for the synthesis of curcumin analogs under microwave conditions, we report herein the microwave-assisted synthesis of curcumin derivatives (1-3) and their cytotoxic activity against breast cancer cell (T47D). During the course of our continuing search for novel curcumin derivatives, we synthesized and characterized of 3,4-Bis-(2hydroxybenzylidene)-piperidin-4-one [10]

\section{EXPERIMENTAL SECTION}

\section{Materials}

Chemicals were 4-methil-piperidone (Merck), 2chloro-benzaldehyde, 3-bromo-benzaldehyde, 4-chlorobenzaldehyde (Aldrich), sodium hydroxide (Merck), and ethanol absolute (Merck).

\section{Instrumentation}

Microwave Mass II (Sineo Microwave Chemistry) was used for the synthesis condition. HPLC (Shimadzu Le Solution), FTIR (Shimadzu, IR Prestige-21), MS (Waters LCT Premier XE ES1-10F) were used for purification of the compounds. ${ }^{1} \mathrm{H}$ and ${ }^{13} \mathrm{C}$-NMR were recorded by using Varian $500 \mathrm{MHz}$.

\section{Procedure}

\section{Synthesis analogues of curcumin}

All the compounds were synthesized by using aldol condensation reaction with sodium hydroxide as catalyst and ethanol as solvent (Carey and Sandberg, 1983). $0.01 \mathrm{~mol} 4$ piperidone derivative was mixed with sodium hydroxide octahydrate $0.7 \mathrm{~mL}(40 \%)$ followed by $10 \mathrm{~mL}$ absolute ethanol in conical flask. Aldehyde derivative (0.02 mol in ethanol $2 \mathrm{~mL}$ ) was added into mixture and finally placed into microwave and the reaction was run for $10 \mathrm{~min}$. Solid product obtained was cooled and $50 \mathrm{~mL} \mathrm{HCl} 1 \mathrm{~N}$ was added subsequently. The mixture was than filtered by using Buchner funnel and washed with $50 \mathrm{~mL}$ distilled water, $50 \mathrm{~mL}$ hexane respectively and finally was dried at $40^{\circ} \mathrm{C}$ for $24 \mathrm{H}$.

\section{RESULT AND DISCUSSION}

\section{$N$-methyl-(3E,5E)-3,5-bis-(2-chlorobenzylidene)-4- piperidone (1)}

Like yellow crystal, $38.90 \%$ yield, melting point: $138-140{ }^{\circ} \mathrm{C}, \mathrm{R}_{\mathrm{f}}=0.67$ ( $n$-hexane : EtOAc $\left.=6: 4\right), \mathrm{HPLC}$, $\mathrm{t}_{R}=16.9 \mathrm{~min}, \mathrm{UV}\left(\lambda_{\max } \mathrm{MeOH}\right): 205 \mathrm{~nm}$ (e 5,600), 239 $\mathrm{nm}(\mathrm{e} 3,800)$ and $310 \mathrm{~nm}$ (e 3,900). IR (KBr) vmax: 3408; 3065; 2975; 1674 and $1620 \mathrm{~cm}^{-1}$. ${ }^{1} \mathrm{H}-\mathrm{NMR}$ $\left(\mathrm{CDCl}_{3}, 500 \mathrm{MHz}\right),{ }^{13} \mathrm{C}-\mathrm{NMR}\left(\mathrm{CDCl}_{3}, 125 \mathrm{MHz}\right)($ Table 2). HR-ESI-TOFMS : $\mathrm{m} / \mathrm{z} \quad 358.0776 \quad[\mathrm{M}+\mathrm{H}]^{+}$, $\mathrm{C}_{20} \mathrm{H}_{17} \mathrm{Cl}_{2} \mathrm{NO} m / z 357.0657$.

Compound 1 was found as $N$-methyl-(3E,5E)-3,5bis-(2-chlorobenzylidene)-4-piperidone with chemical formula $\mathrm{C}_{20} \mathrm{H}_{17} \mathrm{Cl}_{2} \mathrm{NO}$ and molecular weight as 358.0776 $[\mathrm{M}+\mathrm{H}]^{+}$from HR-ESI-TOFMS showed the presence of ion molecule peaks at 20 and $60 \%$ intensity with $\mathrm{m} / \mathrm{z} 361$ and 363 for ${ }^{35} \mathrm{Cl}$ and ${ }^{37} \mathrm{Cl}$. This compound was assigned from ${ }^{1} \mathrm{H}-\mathrm{NMR}$ and ${ }^{13} \mathrm{C}$-NMR (Table 2). From ${ }^{1} \mathrm{H}$-NMR spectra showed the presence of $N$-methyl signal $\left[\delta_{H} 2.37(3 \mathrm{H}, \mathrm{s})\right], 2$ methylene $\left[\delta_{H}\right.$ $3.61(4 \mathrm{H}, \mathrm{s})], 2$ olefinic proton at down field $\left[\delta_{\mathrm{H}} 8.0(2 \mathrm{H}\right.$, s)] and 8 aromatic proton from 2 substituted aromatic ring $\left[\delta_{H} 7.24(2 \mathrm{H}, \mathrm{dd}, J=8.0 ; 1.5 \mathrm{~Hz}), \delta_{H} 7.30(2 \mathrm{H}, \mathrm{d}, J\right.$ $=7.5 \mathrm{~Hz}), \delta_{H} 7.31(2 \mathrm{H}, \mathrm{dd}, J=8.0 ; 1.5 \mathrm{~Hz})$ and $\delta_{H} 7.46$ $(\mathrm{dd}, J=8.0 ; 1.5 \mathrm{~Hz}) .{ }^{13} \mathrm{C}-\mathrm{NMR}$ spectra showed 20 carbon which was consist of 2 methylene carbons ( $\delta_{c}$ $56.7), 1$ conjugated carbon ( $\delta \mathrm{c} 186.1), \mathrm{N}$-methyl signal (ठc 45.5), $10 \mathrm{sp}^{2}$ carbons, and 6 quaternary carbons (Table 2).

The saturated degree point was calculated as 9 out of 12 degrees. The remaining number was matched with tricyclic of symmetrical monoketone curcumin [11]. To determine the moiety position from the compound, HMBC was applied the results showed correlation between olefinic proton at $\delta_{H} 8.0$ with C-4 ( $\left.\delta_{C} 186.1\right)$, C-

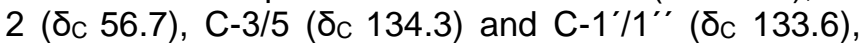
while methylene proton $\left(\delta_{H} 3,61\right)$ showed correlation with C-3/5 ( $\left.\delta_{C} 134.3\right)$ and $C-4\left(\delta_{C}\right.$ 186.1). This result suggested $\alpha, \beta$ unsaturated ketone at $C-7^{\prime}, C-3 / 5$ and C-4.

Chlorine position at both disubstitution aromatic rings was determined from correlation between olefinic

Table 1. Cytotoxic activity against breast cancer cell (T47D)

\begin{tabular}{lcc}
\hline No & Compound & $\mathrm{IC}_{50}(\boldsymbol{\mu g} / \mathbf{m L})$ \\
\hline $\mathbf{1}$ & $N$-metil-(3E,5E)-3,5-bis-(2-klorobenziliden)-4-piperidon (1) & 8 \\
$\mathbf{2}$ & $N$-metil-(3E,5E)-3,5-bis-(3-bromobenziliden)-4-piperidon (2) & 4 \\
$\mathbf{3}$ & $N$-metil-(3E,5E)-3,5-bis-(4-klorobenziliden)-4-piperidon (3) & 45 \\
\hline
\end{tabular}

Yum Eryanti et al. 
Table 2. NMR data of 1, 2 and 3

\begin{tabular}{|c|c|c|c|c|c|c|c|}
\hline \multirow[b]{2}{*}{ No } & \multicolumn{3}{|c|}{1} & \multicolumn{2}{|l|}{2} & \multicolumn{2}{|l|}{3} \\
\hline & Position & ${ }^{1} \mathrm{H}-\mathrm{NMR}$ & $\begin{array}{l}{ }^{13} \mathrm{C}-\mathrm{NMR} \\
\text { (ठc ppm) }\end{array}$ & ${ }^{1} \mathrm{H}-\mathrm{NMR}$ & $\begin{array}{l}{ }^{13} \mathrm{C}-\mathrm{NMR} \\
\text { (ठc ppm) }\end{array}$ & ${ }^{1} \mathrm{H}-\mathrm{NMR}$ & $\begin{array}{l}{ }^{13} \mathrm{C}-\mathrm{NMR} \\
\text { (ठc ppm) }\end{array}$ \\
\hline 1 & $2 / 6$ & $3.61(4 \mathrm{H}, \mathrm{s})$ & 56.7 & $3.73(4 \mathrm{H}, \mathrm{s})$ & 56.8 & $3.71(4 \mathrm{H}, \mathrm{d}, 1.5)$ & 56.9 \\
\hline 2 & $3 / 5$ & - & 134.3 & - & 134.0 & - & 133.4 \\
\hline 3 & 4 & - & 186.1 & - & 186.4 & - & 186.4 \\
\hline 4 & $1 ' / 1 "$ & - & 133.6 & - & 137.2 & - & 133.6 \\
\hline 5 & 2'/2" & - & 135.2 & $7.52(2 \mathrm{H}, \mathrm{s})$ & 132.9 & $7.38(2 \mathrm{H}, \mathrm{d}, 8.5)$ & 128.8 \\
\hline 6 & 3'/3" & $7.24(2 \mathrm{H}, \mathrm{dd}, 7.5 ; 1.5)$ & 130.3 & - & 122.7 & $7.31(2 \mathrm{H}, \mathrm{d}, 8.5)$ & 131.6 \\
\hline 7 & 4'/4" & $7.30(2 \mathrm{H}, \mathrm{d}, 7.5)$ & 130.0 & $7.5(2 \mathrm{H}, \mathrm{dd}, 9.75 ; 2.5)$ & 131.9 & - & 135.1 \\
\hline 8 & $5^{\prime} / 5^{\prime \prime}$ & $7.31(2 \mathrm{H}, \mathrm{dd}, 8.0 ; 1.5)$ & 126.4 & $7.30(2 \mathrm{H}, \mathrm{d}, 6)$ & 130.0 & $7.31(2 \mathrm{H}, \mathrm{d}, 8.5)$ & 131.6 \\
\hline 9 & 6'/6" & $7.46(2 \mathrm{H}, \mathrm{dd}, 8.0 ; 1.5)$ & 129.9 & $7.30(2 \mathrm{H}, \mathrm{d}, 6)$ & 128.8 & $7.38(2 \mathrm{H}, \mathrm{d}, 8.5)$ & 128.8 \\
\hline 10 & 7'/7"' & $8.00(2 \mathrm{H}, \mathrm{s})$ & 134.0 & $7.71(2 \mathrm{H}, \mathrm{s})$ & 135.0 & $7.73(2 \mathrm{H}, \mathrm{s})$ & 135.1 \\
\hline 11 & $\mathrm{~N}-\mathrm{CH}_{3}$ & $2.37(3 \mathrm{H}, \mathrm{s})$ & 45.5 & $2.47(3 \mathrm{H}, \mathrm{s})$ & 45.8 & $2.46(3 \mathrm{H}, \mathrm{s})$ & 45.9 \\
\hline
\end{tabular}

aln $\mathrm{CDCl}_{3}$ at $500 \mathrm{MHz}$ for ${ }^{1} \mathrm{H}-\mathrm{NMR}$ and $125 \mathrm{MHz}$ for ${ }^{13} \mathrm{C}-\mathrm{NMR}$<smiles>[R]c1cccc(/C=C2\CN(C)C/C(=C\c3cccc([R])c3)C2=O)c1</smiles>

Fig 1. The synthesis of $N$-methyl-(3E,5E-bis(R-benzylidene)-4-piperidone<smiles>CN1C/C(=C\c2ccccc2Cl)C(=O)/C(=C/c2ccccc2Cl)C1</smiles>

1<smiles>CN1C/C(=C\c2cccc(Br)c2)C(=O)/C(=C/c2cccc(Br)c2)C1</smiles>

2<smiles>CN1C/C(=C\c2ccc(Cl)cc2)C(=O)/C(=C/c2ccc(Cl)cc2)C1</smiles>

3

Fig 2. Structure of compound 1, 2, and $\mathbf{3}$

proton at $\delta_{H} 8.0$ with $\mathrm{C}-1^{\prime} / 1^{\prime \prime}$ ( $\delta_{C}$ 133.6), C-2'/2" ( $\delta_{C}$ 135.2), aromatic proton at $\delta_{H} 7.3$ with $\mathrm{C}-1^{\prime} / 1^{\prime \prime}\left(\delta_{C}\right.$ 133.6) and $C 5^{\prime} / 5^{\prime \prime}$ ( $\left.\delta_{C} 126.4\right)$, aromatic proton at $\delta_{H} 7.24$

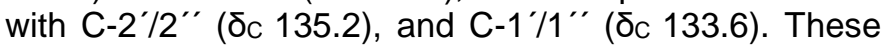
results suggested the chlorine position at ortho position. This suggestion was supported with coupling constant from aromatic proton. Compound 1 structure was confirmed with single X-ray crystal diffraction and ORTEP diagram (Fig. 2.).

\section{$N$-methyl-(3E,5E)-3,5-bis-(3-bromobenzylidene)-4- piperidone (2)}

Like yellow powder, $65.60 \%$ yield, melting point: $129-130{ }^{\circ} \mathrm{C}, \mathrm{R}_{\mathrm{f}}=0.43$ ( $n$-hexane : EtOAc = 7:3), HPLC, $\mathrm{t}_{R}=18.8 \mathrm{~min}$, UV $\left(\lambda_{\max } \mathrm{MeOH}\right): 324 \mathrm{~nm}(\mathrm{e} 3,800)$, $238 \mathrm{~nm}(\mathrm{e} \mathrm{5,600)}$ and $210 \mathrm{~nm}$ (e 5,600). IR (KBr) vmax: 3432; 2927; 1668 and $1613 \mathrm{~cm}^{-1}$. ${ }^{1} \mathrm{H}-\mathrm{NMR}\left(\mathrm{CDCl}_{3}, 500\right.$ $\mathrm{MHz}),{ }^{13} \mathrm{C}-\mathrm{NMR}\left(\mathrm{CDCl}_{3}, 125 \mathrm{MHz}\right)$ (Table 2). HR-ESITOFMS : $m / z \quad 447.9742[\mathrm{M}+\mathrm{H}]^{+}, \quad \mathrm{C}_{20} \mathrm{H}_{17} \mathrm{Br}_{2} \mathrm{NO} \mathrm{m} / \mathrm{z}$ 447.9737 .
Compound 2 was found as $N$-methyl-(3E,5E)-3,5bis-(3-bromobenzylidene)-4-piperidone with chemical formula $\mathrm{C}_{20} \mathrm{H}_{17} \mathrm{Br}_{2} \mathrm{NO}$ and molecular weight as $467.9677[\mathrm{M}+\mathrm{H}]^{+}$from HR-ESI-TOFMS and showed the presence of ion molecule peaks at 45 and $55 \%$ intensity with $\mathrm{m} / \mathrm{z} 445$ and 447 for ${ }^{79} \mathrm{Br}$ and ${ }^{81} \mathrm{Br}$. This compound was assigned from ${ }^{1} \mathrm{H}-\mathrm{NMR}$ and ${ }^{13} \mathrm{C}-\mathrm{NMR}$ (Table 2). From ${ }^{1} \mathrm{H}-\mathrm{NMR}$ spectra showed the presence of $N$-methyl signal $\left[\delta_{\mathrm{H}} 2.47(3 \mathrm{H}, \mathrm{s})\right], 2$ methylene $\left[\delta_{H} 3.73(4 \mathrm{H}, \mathrm{s})\right], 2$ olefinic proton at down field [ $\delta_{H} 7.71$ $(2 \mathrm{H}, \mathrm{s})]$ and 8 aromatic proton from 2 substituted aromatic ring $\left[\delta_{H} 7.52(2 \mathrm{H}, \mathrm{s}), \delta_{H} 7.50(2 \mathrm{H}, \mathrm{dd}, J=9.7\right.$; $2.5 \mathrm{~Hz}), \delta_{\mathrm{H}} 7.30(2 \mathrm{H}, \mathrm{dd}, J=6 \mathrm{~Hz})$ and $\delta_{\mathrm{H}} 7.46(\mathrm{dd}$, $J=8.0 ; 1.5 \mathrm{~Hz}) .{ }^{13} \mathrm{C}-\mathrm{NMR}$ spectra showed 20 carbon which was consist of 2 methylene carbons ( $\delta_{c} 56.7$ ), 1 conjugated carbon ( $\delta \mathrm{c}$ 186.4), $N$-methyl signal ( $\left.\delta_{c} 45.8\right)$, and $16 \mathrm{sp}^{2}$ carbons (Table 2).

The saturated degree point was calculated as 9 out of 12 degrees. The remaining number was matched with tricyclic of symmetrical monoketone curcumin [11]. The bromine at both aromatic rings was determined 


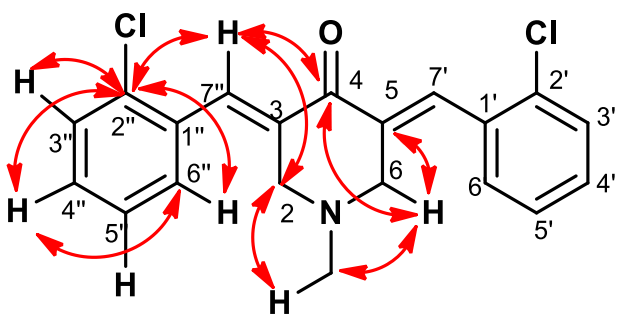

Fig 3. HMBC correlation of compound 1

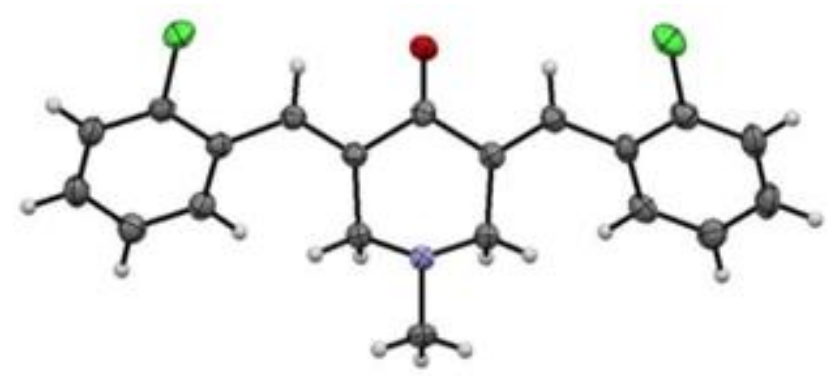

Fig 4. ORTEP of compound 1

based on coupling constant as meta position. The comparison of NMR data from compound 2 with $\mathrm{N}$ methyl-(3E,5E)-3,5-bis-(3-bromobenziliden)-4-piperidone [11-13] showed similarity.

\section{$N$-methyl-(3E,5E)-3,5-bis-(4-chlorobenzylidene)-4- piperidone (3)}

Yellow crystal, $39.80 \%$ yield, melting point: 180 $182{ }^{\circ} \mathrm{C}, \mathrm{R}_{f}=0.40$ ( $n$-hexane : EtOAc = 8:2), HPLC, $\mathrm{t}_{R}=17.8 \mathrm{~min}$, UV $\left(\lambda_{\max } \mathrm{MeOH}\right): 202 \mathrm{~nm}($ e 5,600), $236 \mathrm{~nm}(\mathrm{e} 5,600)$ and $333 \mathrm{~nm}$ (e 5,600). IR (KBr) vmax: 3659; 3432; 2936; 1672 and $1613 \mathrm{~cm}^{-1}$. ${ }^{1} \mathrm{H}-\mathrm{NMR}\left(\mathrm{CDCl}_{3}\right.$, $500 \mathrm{MHz}),{ }^{13} \mathrm{C}-\mathrm{NMR}\left(\mathrm{CDCl}_{3}, 125 \mathrm{MHz}\right)$ (Table 2). HRESI-TOFMS : $\mathrm{m} / \mathrm{z} 358.0776[\mathrm{M}+\mathrm{H}]^{+}, \mathrm{C}_{20} \mathrm{H}_{17} \mathrm{Cl}_{2} \mathrm{NO} \mathrm{m} / \mathrm{z}$ 357.0687.

Compound 3 was found as $N$-methyl-(3E,5E)-3,5bis-(4-chlorobenzylidene)-4-piperidone with chemical formula $\mathrm{C}_{20} \mathrm{H}_{17} \mathrm{Cl}_{2} \mathrm{NO}$ and molecular weight as 358.0776 $[\mathrm{M}+\mathrm{H}]^{+}$from HR-ESI-TOFMS showed the presence of ion molecule peaks at 20 and $60 \%$ intensity with $\mathrm{m} / \mathrm{z} 361$ and 363 for ${ }^{35} \mathrm{Cl}$ and ${ }^{37} \mathrm{Cl}$. The comparison NMR spectra from compound $\mathbf{3}$ and $\mathbf{1}$ showed the similarity. The para position of chlorine in compound $\mathbf{3}$ was assigned from proton NMR at aromatic proton $\left[\delta_{\mathrm{H}} 7.31(4 \mathrm{H}, \mathrm{d}, J=8.5\right.$ $\mathrm{Hz})$ and $\left.\delta_{H} 7.38(4 \mathrm{H}, \mathrm{d}, J=8.5 \mathrm{~Hz})\right]$.

\section{Cytotoxic Activity against Breast Cancer Cell Line (T47D)}

All the compounds were screened their cytotoxic activity against breast cancer cell line (T47D) by using MTT assay [18]. The $\mathrm{IC}_{50}$ showed that 2 possessed high activities $(4 \mu \mathrm{g} / \mathrm{mL})$ compared with 1 and 3 with value 6 and $45 \mu \mathrm{g} / \mathrm{mL}$ (Table 1) respectively. It might be due to the electronegativity of halogen and the position of halogen where meta position was more active than ortho and para.

\section{CONCLUSION}

Three piperidone curcumin analogues have been synthesized by using aldol condensation reaction with base catalyst and irradiation from microwave. Compound 2 showed high cytotoxic activities compared to another compounds against breast cancer cell line (T47D).

\section{ACKNOWLEDGEMENT}

This research was partially supported by Directorate General of Higher Education, Ministry of Education and Culture, Indonesia.

\section{REFERENCES}

[1] Rostom, S.A., Hassan, G.S., and El-Subbagh, H.I., 2009 , Synthesis and biological evaluation of some polymethoxylated fused pyridine ring systems as antitumor agents, Arch. Pharm., 342 (10), 484590.

[2] Dandia, A., Jain, A.K., and Sharma, S., 2012, An efficient and highly selective approach for the construction of novel dispiro heterocycles in guanidine-based task-specific [TMG][Ac] ionic liquid, Tetrahedron Lett., 53 (44), 5859-5863.

[3] Kumar, R.R., Perumal, S., Senthilkumar, P., Yogeeswari, P., and Sriram, D., 2007, An atom efficient, solvent-free, green synthesis and antimycobacterial evaluation of 2-amino-6-methyl4-aryl-8-[( $E$-arylmethylidene]-5,6,7,8-tetrahydro4H-pyrano[3,2-c]pyridine-3-carbonitriles, Bioorg. Med. Chem. Lett., 17 (23), 6459-6462.

[4] Suzuki, M., Nakamura, T., Iyoki, S., Fujiwara, A., Watanabe, Y., Mohri, K., Isobe, K., Ono, K., and Yano, S., 2005, Elucidation of anti-allergic activities of curcumin-related compounds with a special reference to their anti-oxidative activities, Biol. Pharm. Bull., 28 (8) 1438-1443.

[5] Handler, N., Jaeger, W., Puschacher, H., Leisser, K., and Erker, T., 2007, Synthesis of novel curcumin analogues and their evaluation as selective cyclooxygenase-1 (COX-1) inhibitors, Chem. Pharm. Bull., 55 (1), 64-71.

[6] Yadav, B., Taurin, S., Rosengren, R.J., Schumacher, M., Diederich, M., Somers-Edgar, T.J., and Larsen, L., 2010, Synthesis and cytotoxic potential of heterocyclic cyclohexanone analogues 
of curcumin, Bioorg. Med. Chem., 18 (18), 67016707.

[7] Wichitnithad, W., Nimmannit, U., Wacharasindhu, S., and Rojsitthisak, P., 2011, Synthesis, characterization and biological evaluation of succinate prodrugs of curcuminoids for colon cancer treatment, Molecules, 16 (2), 1888-1900.

[8] Subramaniam, D., Nicholes, N.D., Dhar, A., Umar, S., Awasthi, V., Welch, D.R., Jensen, R.A., and Anant, S., 2011, 3,5-bis(2,4-difluorobenzylidene)-4piperidone, a novel compound that affects pancreatic cancer growth and angiogenesis, Mol. Cancer Ther., 10 (11), 2146-2156.

[9] Lagisetty, P., Powell, D.R., and Awasthi, V., 2009, Synthesis and structural determination of 3,5-bis(2fluorobenzylidene)-4-piperidone analogs of curcumin, J. Mol. Struct., 936 (1-3), 23-28.

[10] Eryanti, Y., Herlina, T., Zamri, A., Halim, S.N.A., Shiono, Y., Syah, Y.M., Awang, K., and Supratman, U., 2014, 3,5-Bis(2-hydroxybenzylidene)piperidin-4one, Molbank, M825, 1-3.

[11] Gregory, M., Dandavati, A., Lee, M., Tzou, S., Savagian, M., Brien, K.A., Satam, V., Patil, P., and Lee, M., 2013, Synthesis, cytotoxicity, and structure-activity insight of $\mathrm{NH}$ - and $\mathrm{N}$-methyl-3,5bis-(arylidenyl)-4-piperidones, Med. Chem. Res., 22 (11), 5588-5597.

[12] Mohamed, A.M., El-Sayed, W.A., Alsharari, M.A., Al-Qalawi, H.R., and Germoush, M.O., 2013, Anticancer activities of some newly synthesized pyrazole and pyrimidine derivatives, Arch. Pharm. Res., 36 (9),1055-1065.
[13] Das, U., Singh, R.S.P., Alcorn, J., Hickman, M.R., Sciotti, R.J., Leed, S.E., Lee, P.J., Roncal, N., and Dimmock, J.R., 2013, 3,5-Bis(benzylidene)-4piperidones and related $\mathrm{N}$-acyl analogs: A novel cluster of antimalarials targeting the liver stage of Plasmodium falciparum, Bioorg. Med. Chem., 21 (23), 7250-7256.

[14] Kelkel, M., Jacob, C., Dicato, M., and Diederich, M., 2010, Potential of the dietary antioxidants resveratrol and curcumin in prevention and treatment of hematologic malignancies, Molecules, 15, 7035-7074.

[15] Shimatsu, A., Kakeya, H., Imaizumi, A., Morimoto, T., Kanai, M., and Maeda, S., 2012, Clinical application of "curcumin", a multi-functional substance, Anti-Aging Med., 9 (2), 75-83.

[16] Eryanti, Y., Zamri, A., Frimayanti, N., Supratman, U., and Herlina, T., 2016. Dataset of curcumin deivatives of QSAR modeling of anticancer against P388 cell line, Data Brief, 9, 573-578.

[17] Eryanti, Y., Zamri, A., Supratman, U., Herlina, T., Rosli, M.M., and Fun, H.K., 2015, Crystal structures of 3,5-bis[(E)-3-hydroxybenzylidene]-1methylpiperidin-4-one and 3,5-bis[(E)-2chlorobenzylidene]-1-methylpiperidin-4-one, Acta Cryst., E71,1488-1492.

[18] Alley, M.C., Scudiero, D.A., Monks, A., Hursey, M.L., Czerwinski, M.J., Fine, D.L., Abbott, B.J., Mayo, J.G., Shoemaker, R.H., and Boyd, M.R., 1988, Feasibility of drug screening with panels of human tumor cell lines using a microculture tetrazolium assay, Cancer Res., 48 (3), 589-601 